\title{
Rolling Rod Electrostatic Microgenerator
}

\author{
Michail E. Kiziroglou, Member IEEE, Cairan He and Eric M. Yeatman, Senior Member, IEEE
}

\begin{abstract}
The difficulty of maximizing the proof mass, and lack of broadband operation, are key issues for miniaturized energy harvesting devices. Here, a novel electrostatic energy harvester is presented, employing an external, free-rolling proof mass to address these issues. A description of the operating principle is given and the kinetic dynamics of the cylinder are analyzed. The electrostatics of the system are simulated, identifying the device performance for different dielectric dimensions and surface specifications. The fabrication of a prototype device is presented and physical characterization results demonstrate a successful fabrication technique for dielectric sizes down to $100 \mathrm{~nm}$. Capacitance measurements reveal a capacitance ratio of 4 and are in agreement with simulation results. A voltage gain of 2.4 is demonstrated. The device is suitable for energy harvesting from low frequency, high amplitude ambient motion sources, such as the human body.
\end{abstract}

Index Terms - electrostatic, energy harvesting, MEMS, power scavenging

\section{INTRODUCTION}

E nergy harvesting devices are attractive for applications where battery replacement is impractical, either because of position inaccessibility, a large number of devices, or both. Such applications include body mounted medical sensors and implantable devices, wireless sensor networks, structural and field condition monitoring and sensors in vehicle tyres. In many of these cases, light is not reliably available and temperature gradients are modest, making ambient motion a more suitable source of power.

The low frequencies of body motion offer limited power densities [1], and since a high degree of miniaturization is desirable, achieving near-optimal designs will be necessary for these applications to be feasible. A wide range of motion scavenging devices has been reported [2]; most are based on a proof mass mounted to a frame with a flexible suspension, with power generated by damping the relative proof mass motion with a transducer element.

While electromagnetic forces are most suitable for energy conversion at macroscopic dimensions, scaling laws make electrostatic forces more feasible for energy conversion at the

Manuscript received April 15, 2008. Accepted for publication July 29, 2008.

Copyright (c) 2007 IEEE. Personal use of this material is permitted. However, permission to use this material for any other purposes must be obtained from the IEEE by sending a request to pubs-permissions@ieee.org.

M. E. Kiziroglou, C. He and E. M. Yeatman are with the optical and semiconductor devices group, Imperial College London, SW7 2BT, U.K. (phone: 0044207594 6216; fax: 0044207594 6308; e-mail: m.kiziroglou@ic.ac.uk). $\mathrm{mm}$ scale and below [3]. Electrostatic devices are also convenient for integrated manufacturing, as they do not require the specialized materials and associated fabrication processes of magnetic and piezoelectric systems. For these reasons, electrostatic transduction has been investigated by a number of groups for use in miniature energy harvesting devices, particularly ones implemented in silicon Micro-ElectroMechanical Systems (MEMS).

The first reports were by Meninger et al. [4], and describe a monolithic MEMS device with a laterally moving proof mass, and comb drive electrostatic transducers. The relative merits of constant charge and constant voltage operation were analyzed. However, successful net power generation from these devices was not reported. Similar devices were also investigated by Roundy et al. [5], who also provide the first report of electrostatic harvesters with integrated output electronics. Generally electrostatic devices have the disadvantage that a precharge or priming voltage must be applied on each cycle, but this can be avoided with the use of integrated electrets. Such electret-based harvesters have been reported by Sterken et al. [6].

Successful operation of a non-resonant MEMS device suitable for low frequency applications, such as body motion, has been reported by Miao et al. [7]. More recently Yen and Lang have reported net power of $1.8 \mu \mathrm{W}$ from an electrostatic device which incorporates an inductive energy flyback circuit to provide the priming energy [8].

In general, MEMS electrostatic energy harvesters have an integrated mass forming one element of a variable capacitor, with power derived from an electrostatic force between the moving mass and the frame [9]. Such a monolithic device is attractive for size and (potentially) cost reasons, but has several important limitations. Firstly, maximizing the proof mass necessitates machining through the full thickness of the wafer, which is incompatible with most integrated electronics processes. MEMS inertial sensors with monolithically integrated electronics are well established commercially, and are similar in structure to inertial energy harvesters, but typically use surface mounted proof masses of inadequate size for energy generation use. Furthermore, obtaining a substantial travel range with a monolithic device is difficult, as is a suspension which is highly flexible in the travel direction but effectively prevents motion in other axes.

Devices in which the proof mass is fixed using a stiff suspension form a mechanically resonant system. This is beneficial where the source motion amplitude is small, as it can provide resonant enhancement of the internal motion range, but has the important limitation of requiring excitation at or near a particular resonant frequency. This is especially inappropriate for body mounted energy harvesters, given the 
broad and varying spectrum of body motion. A possible solution to this problem is to actively tune the resonance frequency. Such tuning will typically require active control of the suspension stiffness; for example, in [10] a device is investigated in which permanent magnets provide additional stiffness that can be varied by adjusting the position of the magnets.

In this paper a novel electrostatic energy harvester is described. An external, free-rolling proof mass is used to address both the requirements for a large mass and broadband operation. All the other components are monolithically integrated with standard surface processing. We have previously presented this device concept in [11], but problems of inadequate surface flatness, low effective device capacitance and excessive parasitic capacitance to the substrate meant that useful output power was not obtained. Here we present a revised design and process flow, and show that output power is achieved.

\section{OPERATING PRINCIPLE}

The operating principle is illustrated in Fig. 1. A series of strip electrodes form the fixed plates of the variable capacitor, and are covered by a thin dielectric layer. A metal cylinder forms the moving counter-electrode. When the cylinder is aligned with one of the strip electrodes, it makes direct contact with an additional (narrow) charging contact, by which the necessary pre-charge is applied. This creates an electrostatic force between the cylinder and the strip electrode. Motion of the substrate then induces rolling of the cylinder, causing it to break contact with the pre-charge supply; the separation is then increased at constant charge, so that as the capacitance reduces, the stored energy increases due to the work done against the electrostatic force. The cylinder then makes contact with a discharge electrode, releasing this energy in the form of a high voltage pulse. This operation is equivalent to that of the monolithic device of [7], but has several key advantages: the mass can be significantly greater for an equivalent device size; no suspension structure is needed (although a guide structure will be required to constrain the motion); the travel range (to which the achievable power is also proportional) is greatly increased, partly because of the elimination of the flexural suspension; and the output is provided in several pulses per motion cycle, rather than a single one. The latter characteristic is valuable because parasitic capacitances typically make it difficult to benefit from a large motion range in a single pulse system.

\section{DYNAMIC ANALYSIS}

The motion of the rod while the platform undergoes horizontal motion can be classified into slip and nonslip motion. The nonslip response can be analyzed using the notation of Fig. 2. We take $y(t)$ and $x(t)$ as the absolute positions of the platform and rod, with $z(t)$ the relative motion, i.e. $z(t)=x(t)-y(t)$.

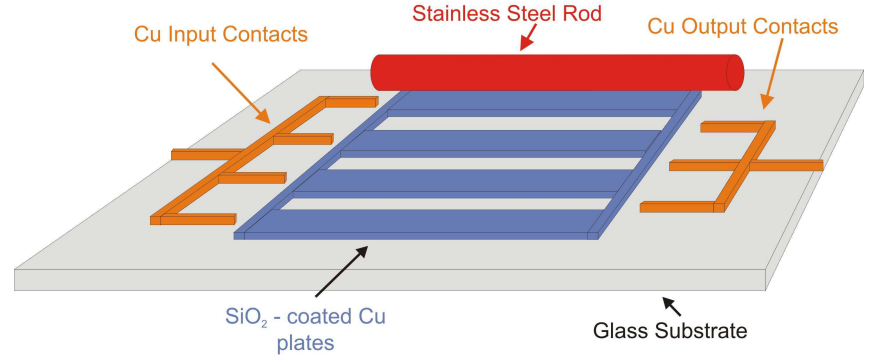

(a)

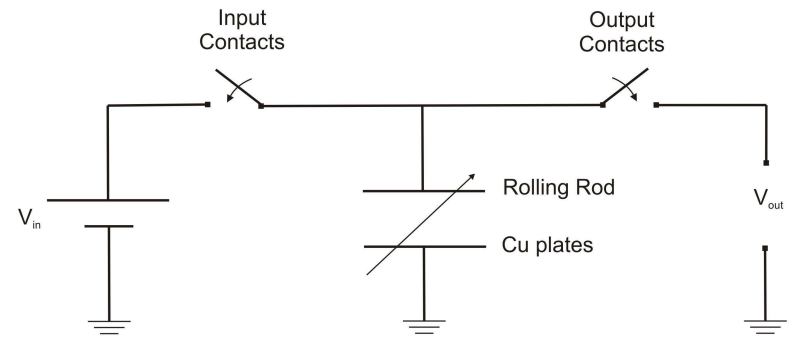

(b)

Fig. 1: (a) Schematic of the device structure; (b) Schematic circuit showing electrical operation.
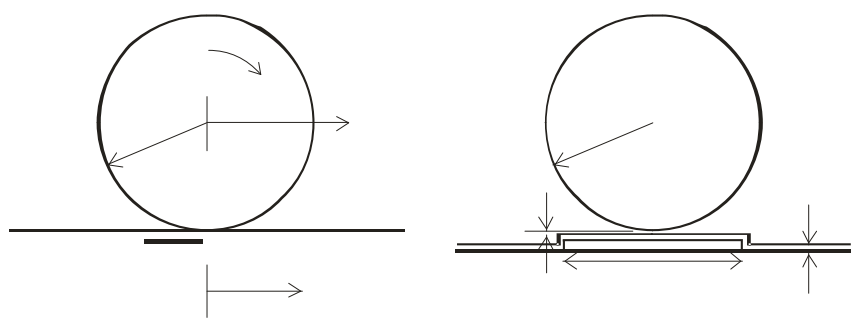

Fig. 2: Geometric notation for dynamic motion analysis $(l)$, and device structure notation $(r)$.

We can then write $z(t)=R \theta(t)$, and thus:

$$
\theta=\frac{x}{R}-\frac{y}{R}
$$

The platform motion will cause a force $F$ to be applied on the rod at the point of contact, which accelerates the rod according to $F=m \ddot{x}$, with $\ddot{x}$ the second time derivative of $\mathrm{x}$. The force also results in a torque which rotates the rod according to $F R=I \ddot{\theta}$, with $I$ the moment of inertia. Taking the second derivative of (1) and equating $\ddot{\theta}$ gives:

$$
\frac{\ddot{x}}{R}-\frac{\ddot{y}}{R}=-\frac{m \ddot{x} R}{I}
$$

which can be manipulated to give:

$$
\left.\ddot{y}=\ddot{x}\left(1+m R^{2} / I\right)\right)
$$

For a solid $\operatorname{rod}, I=m R^{2} / 2$. If we assume harmonic motion and integrate (3) we then get $x(t)=y(t) / 3$, or equivalently

$$
z(t)=-\frac{2}{3} y(t)
$$


This result is independent of frequency, indicating the broadband nature of the response. However, it does show that large source displacement amplitudes are needed to achieve the full internal travel range. Note that in this analysis the electrostatic forces are not included, and these will tend to impede the relative motion; the full analysis is reported in detail for linear motion electrostatic devices in [9].

When sliding happens, the analysis becomes more complex. The condition for sliding to occur is when the maximum static friction is no longer able to provide enough acceleration to synchronize the rod with the platform, i.e. for a harmonic source motion $y(t)=Y_{o} \cos (\omega t)$, sliding occurs if:

$$
\omega_{0} \geq \sqrt{\frac{3 \mu g}{Y_{0}}}
$$

A comparison between simulated nonslip and slip motion is shown in Fig. 3.

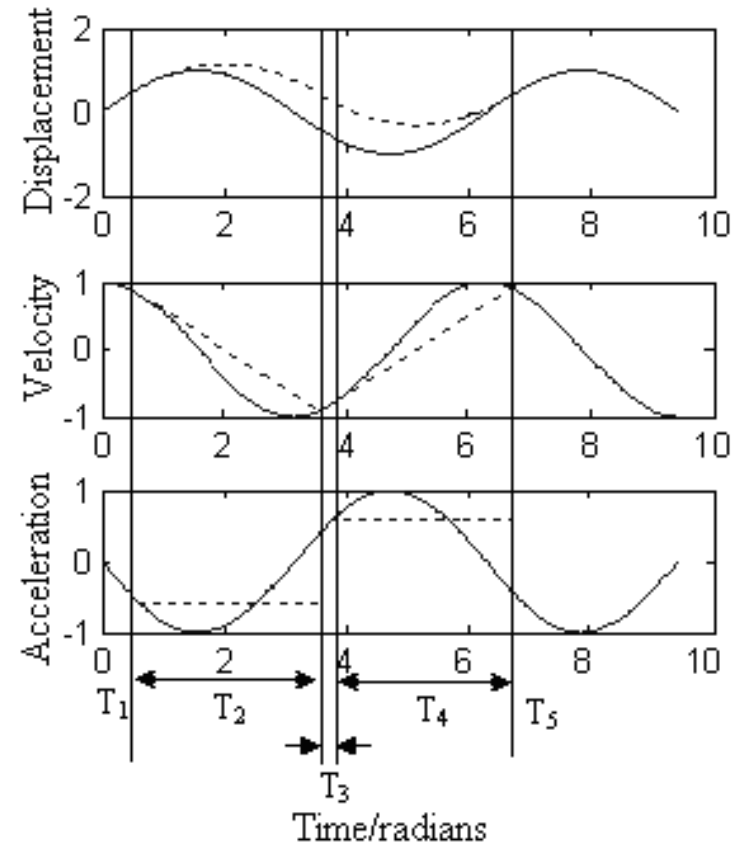

Fig. 3: Dynamic analysis of a rolling cylinder on a plain surface, for nonslip (solid line) or slip (dashed line) motion.

During $T_{1}$ the two motions coincide. At the start of $T_{2}$ the acceleration of the platform exceeds the condition in (5) and sliding starts. During this time the acceleration of the rod will be constant because of constant sliding friction, velocity varies linearly because of constant acceleration, and displacement is quadratic. The two become in phase (no sliding) again when the velocity of the rod is the same as the platform, and the acceleration required at that time can be sufficiently provided by the static friction $\left(T_{3}\right)$. At the end of $T_{3}$, the platform acceleration again exceeds friction's capability, and sliding starts. In $T_{4}$ the rod moves as in $T_{2}$ but in the opposite direction, and $T_{5}$ is a repetition of $T_{1}$. Sliding occurs in $T_{2}$ and $T_{4}$; in other time slots only rotation takes place.

\section{Simulations}

The energy extracted per charge-discharge cycle in a constant charge electrostatic generator is given by:

$$
\Delta U=\frac{1}{2} \frac{C_{\max }}{C_{\min }}\left(C_{\max }-C_{\min }\right) V_{i}^{2}
$$

where $C_{\max }$ and $C_{\min }$ are the initial and final capacitances, and $V_{i}$ the priming voltage. Thus it is important to maximize both the capacitance difference and the ratio, and since the minimum useable level will be limited by parasitics, maximizing $C_{\max }$ is crucial. The capacitance between a cylinder and a flat plane can be solved analytically. However, here we have the added complications of a finite lower electrode width $w$, and a flat dielectric of thickness $\tau$ and relative permittivity $\varepsilon_{r}$, while the rest of the dielectric is air. There is also the possibility of a small air gap $g$ between the rod and the dielectric film, for example because of rod or substrate bow. Thus we have used finite element simulation to determine the capacitance. The results are shown below.

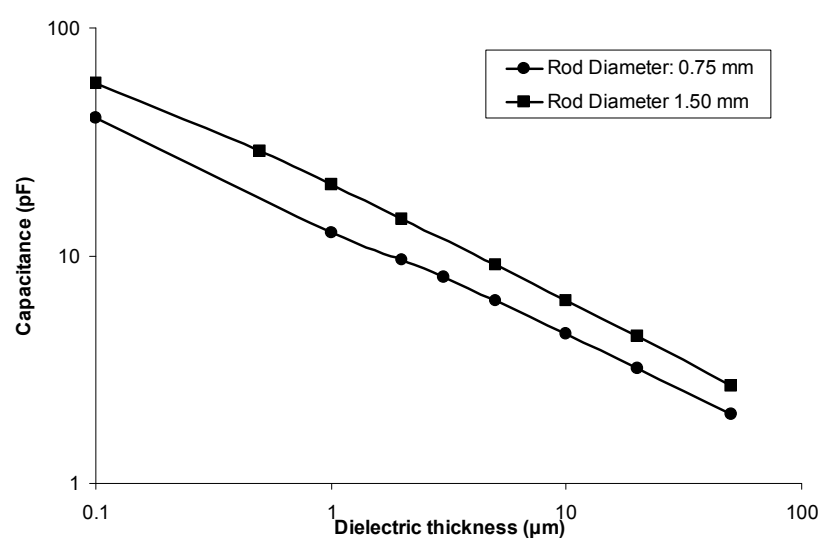

Fig. 4: Rod/Plate capacitance versus dielectric thickness for two different rod diameters. The plate length was $10 \mathrm{~mm}$ and the plate width $1 \mathrm{~mm}$.

Fig. 4 shows the capacitance versus dielectric thickness for different rod radii. Clearly obtaining a low dielectric thickness is required, and this must be achieved over a significant surface area while avoiding pinholes that could lead to device shorting. We chose sputtered $\mathrm{SiO}_{2}$ as our material, for its high deposited quality and stability, and good thickness control.

It is also possible that the rod does not achieve intimate contact with the dielectric layer, for example because of surface roughness or curvature, and so we have simulated the effect of a small air gap. The results (Fig. 5) indicate that the gap must be less than a tenth the oxide thickness in order to have negligible effect.

\section{FABRICATION}

The wafer-lever fabrication process for the device is shown in Fig. 6. 


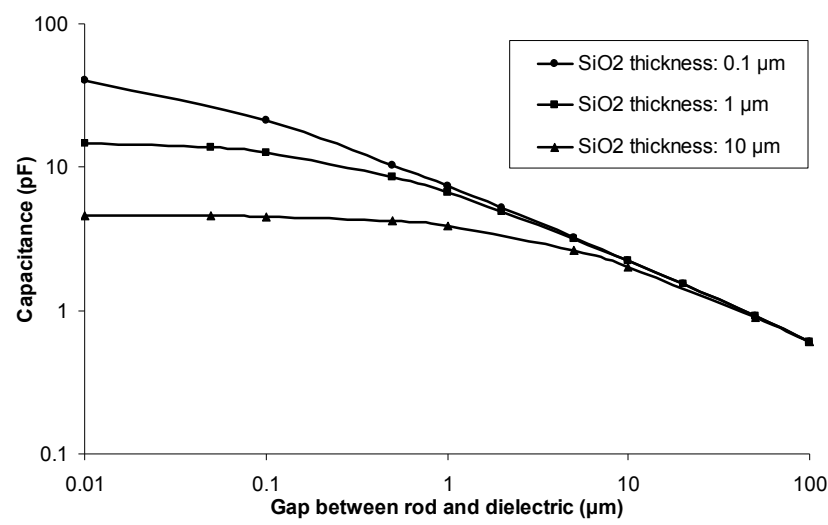

Fig. 5: Capacitance versus size of gap between rod and dielectric, for different dielectric thicknesses. The plate length was $10 \mathrm{~mm}$ and the plate width $1 \mathrm{~mm}$.
Starting material: Glass

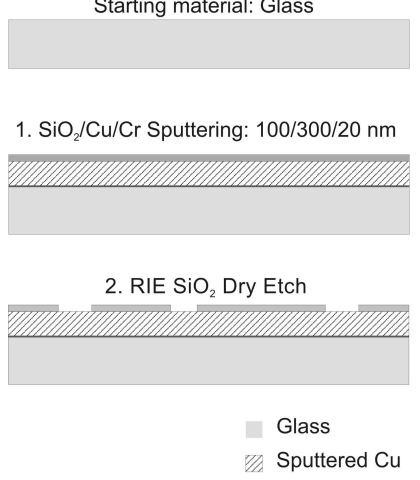

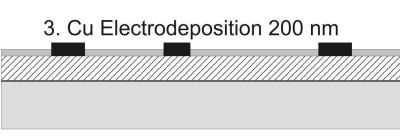
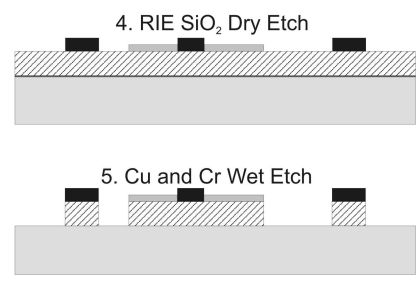

Sputtered $\mathrm{SiO}_{2}$

- Electrodeposited Cu
Fig. 6: Fabrication process flow for the substrate of the generator.

A 4-inch, $500 \mu \mathrm{m}$ thick Schott Borofloat33 glass wafer was used as the substrate; glass is used rather than $\mathrm{Si}$ in order to minimise parasitic capacitances. A $20 \mathrm{~nm}$ thin $\mathrm{Cr}$ adhesion layer and a $300 \mathrm{~nm} \mathrm{Cu}$ layer were sputtered on the glass wafer, followed by $100 \mathrm{~nm} \mathrm{SiO}_{2}$ sputtering (step 1 in Fig. 6). By conventional lithography, patterns of photoresist were defined on top, exposing only the contact areas of the devices. The $\mathrm{SiO}_{2}$ was etched at the contact areas using reactive ion etching (RIE) (step 2), and $\mathrm{Cu}$ electrodeposition followed, to a thickness of $200 \mathrm{~nm}$ (step 3). This technique is promising for much finer calibration at the edge between the $\mathrm{SiO}_{2}$ and the $\mathrm{Cu}$ contact. Subsequently, the resist was stripped off the wafer and a new resist pattern was defined to cover the plate and contact regions of the device. The $\mathrm{SiO}_{2}$ was etched using RIE (step 4) and the $\mathrm{Cu}$ and $\mathrm{Cr}$ were etched using appropriate wet chemical etchants (step 5). A steel rod with diameter $0.75 \mathrm{~mm}$ was used for the proof mass.

An optical image of the device is shown in Fig. 7. The glass substrate with the $\mathrm{Cu}$ contacts and the $\mathrm{SiO}_{2}$ covered $\mathrm{Cu}$ electrode strips is visible. The width of the strips is $1 \mathrm{~mm}$ and the space between them is $1.75 \mathrm{~mm}$. The length of the strips is $8 \mathrm{~mm}$. A steel rod is also shown, with a diameter of $0.75 \mathrm{~mm}$ and length $10 \mathrm{~mm}$. In the inset, a whole glass wafer of devices is illustrated.

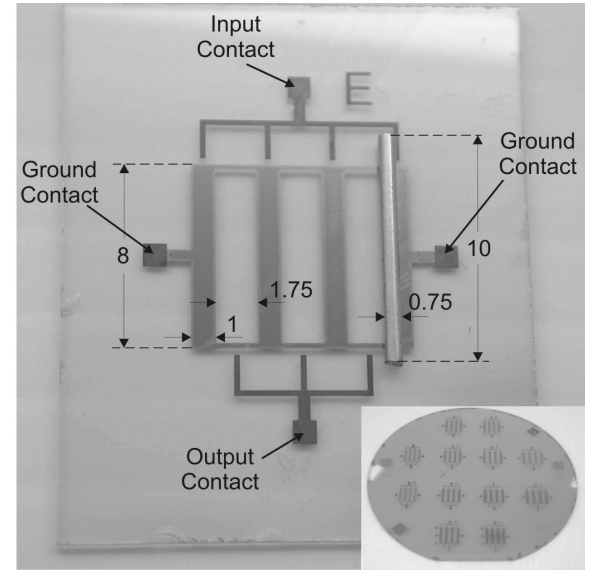

Fig. 7: Optical image of a fabricated harvesting device including the stainless steel rod. The noted dimensions are in millimeters. A whole wafer of devices is shown in the inset.

\section{CHARACTERIZATION}

\section{A. Physical characterization}

Optical interferometry measurements were performed using a Zygo interferometer, for an accurate determination of the physical dimensions of the device. A cross-section of the device was extracted from the interferometry measurements along the dashed line of Fig. 8. The resulting profile is plotted in Fig. 9. The thicknesses of the $\mathrm{SiO}_{2}$ - covered plates and the contacts are 400 and $650 \mathrm{~nm}$ respectively, giving a step of 250 $\mathrm{nm}$. This step ensures that the rolling rod makes contact with the input and the output pads as it rolls over the isolated plates. The steps marked as " $A$ " in Fig. 9 are image artefacts resulting from stitching of sub-fields during interferometry scanning. The root mean square surface roughnesses of the sputtered $\mathrm{SiO}_{2}$ and the electrodeposited $\mathrm{Cu}$ are both less than $10 \mathrm{~nm}$. For these measurements a square area with side $0.8 \mathrm{~mm}$ was used.

The corresponding surface profiles are shown in Fig. 10. These indicate a roughness amplitude of only a few nm.

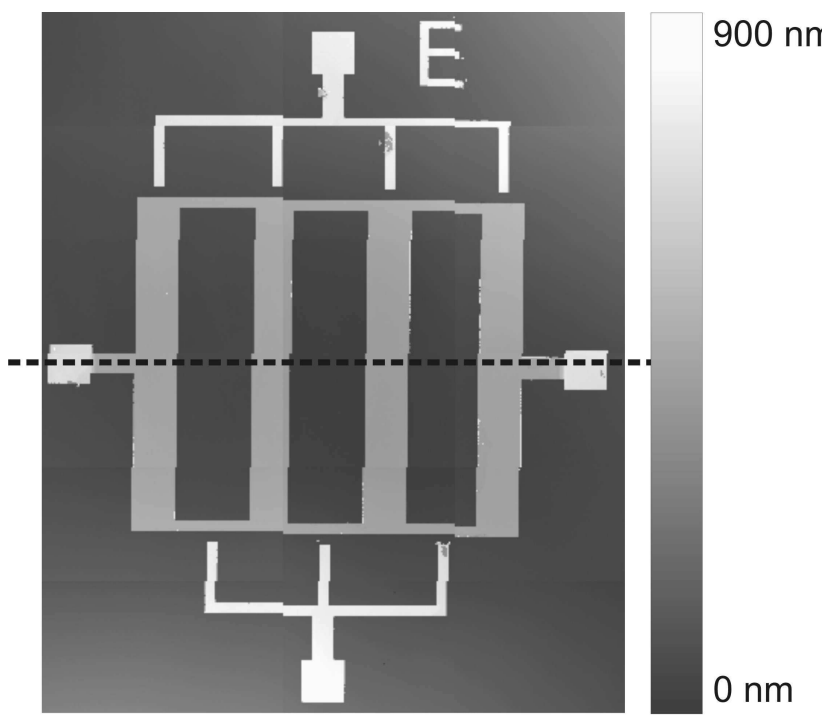

Fig. 8: Optical interferometry measurements. 


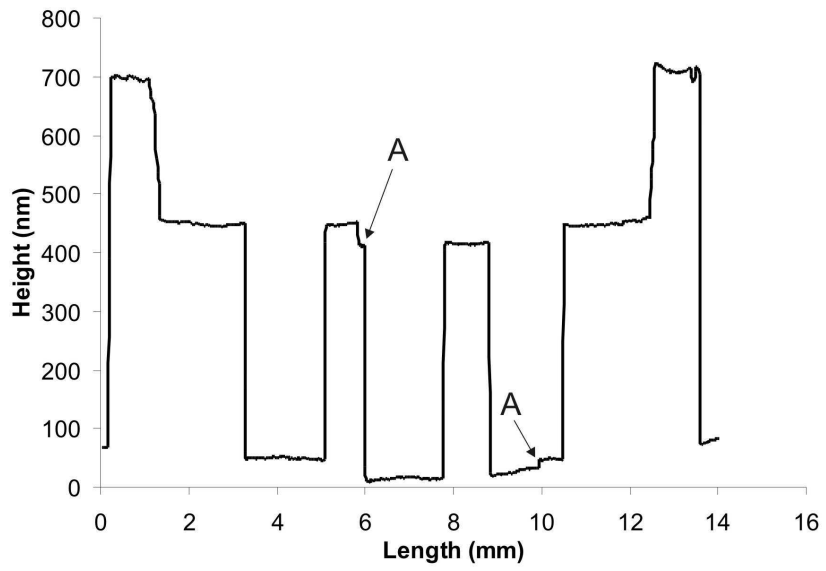

Fig. 9: Cross section profile, along the line indicated in Fig. 8. The plates and the contact thicknesses are around $400 \mathrm{~nm}$ and $650 \mathrm{~nm}$ respectively.
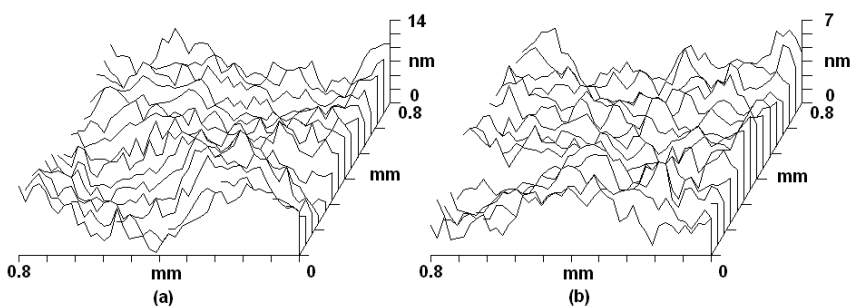

Fig. 10: Surface profiles (a) for sputtered $\mathrm{SiO}_{2}$ and (b) electrodeposited $\mathrm{Cu}$.

\section{B. Capacitance Measurements}

Among the most critical parameters for the successful operation of an electrostatic generator are the useful and the parasitic capacitances throughout the device structure. The parasitic capacitances among the input contacts, the plates and the output contacts were below the limits of detection of our apparatus at about $1.5 \mathrm{pF}$.

The capacitance between the steel rod and the plates was measured for different positions of the rod on the substrate along $1.25 \mathrm{~mm}$ from either side of the centre of a substrate plate. During measurements, the rod was parallel to the long direction of the plates. The resulting values are plotted as black dots against displacement in Fig. 11. At the discharging position, a capacitance of around $2 \mathrm{pF}$ is observed. As the rod reaches the left side of the plate, a sudden increase of the capacitance to around $8 \mathrm{pF}$ is observed. The high capacitance value remains as long as the rod is above the plate. This corresponds to the charging position. When the rod rolls off the plate, the capacitance drops back to the value of around 2 $\mathrm{pF}$.

Simulations of the capacitance against rod displacement of this geometry have been performed using the ANSYS software package. Assuming perfect contact of the rod to the dielectric, a rod-to-plate capacitance value of $40 \mathrm{pF}$ is expected at the charging position, for the geometric parameter values in this device. The difference between the measured and the calculated rod-to-plate capacitance can be explained by taking into account the imperfection of rod-to-dielectric contact. If an effective air gap of $1 \mu \mathrm{m}$ is assumed, simulations give a capacitance of around $6 \mathrm{pF}$. The corresponding simulation results are shown as a solid line in Fig. 11.

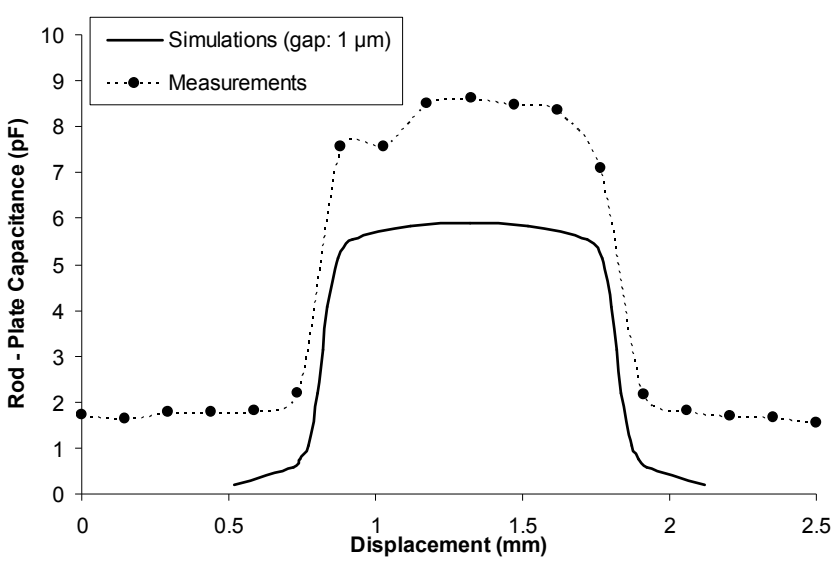

Fig. 11: Capacitance between rod and plate versus displacement, as the rod rolls over one of the $\mathrm{SiO}_{2}$ covered plates. The displacement is measured from the middle of the gap between two plates (see Fig. 7).

The experimental behaviour of capacitance versus lateral displacement matches the one predicted by the numerical simulations. The excess capacitance of around $2 \mathrm{pF}$ observed throughout the displacement measurement range corresponds to the parasitic capacitance of the electrodes and measuring equipment. This matching indicates a $1 \mu \mathrm{m}$ effective gap between rod and dielectric. This can be explained by taking into account the large length of the rod to substrate contact (10 $\mathrm{mm}$ ), for which deviations on the micrometer scale can be explained by very modest bowing $(1 \mu \mathrm{m}$ corresponding to a radius of curvature of $10 \mathrm{~m}$ )

The in-plane electrostatic force applied on the rod can be written as a function of the stored charge $Q$, the capacitance $C$ and the capacitance gradient with respect to the displacement $\mathrm{x}$ :

$$
F=-\frac{d E_{C}}{d x}=\frac{Q^{2}}{2} \cdot \frac{1}{C^{2}} \cdot \frac{d C}{d x}
$$

where $E_{C}$ is the electrostatic energy stored in the device. The direction of the force is such that the rod is attracted towards alignment with the closest plate.

Using the simulation results of Fig. 11 for the rod - plate capacitance, the force as a function of displacement is calculated. The results are plotted in Fig. 12. While the rod is on the $\mathrm{SiO}_{2}$ covered plate (displacement $1.25 \pm 0.5 \mathrm{~mm}$ in Fig. 12), the in-plane force is negligible. As the rod rolls off the plate there is a sharp increase of the absolute value of the force. Subsequently, as the rod rolls away from the plate, a constant electrostatic force is expected, for distances up to 1 $\mathrm{mm}$ from the plate. At the discharge contacts, the charge is removed, so the force drops to zero until the rod reaches the next charging contact. 


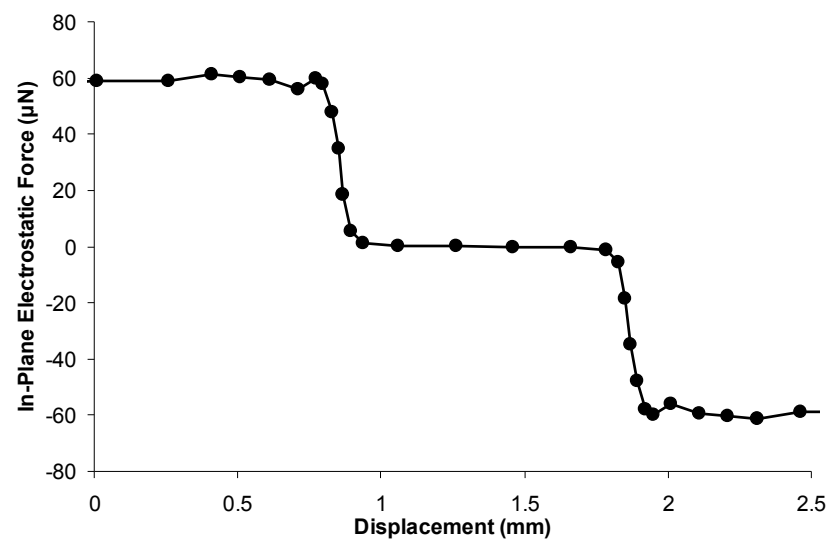

Fig. 12: Simulated electrostatic force between rod and plate versus displacement. The displacement is measured from the middle of the gap between two plates (see Fig. 7)

\section{Output Voltage Measurements}

The operation of the fabricated generator was tested by measuring the output voltage while a $0.75 \mathrm{~mm}$ diameter rod was rolling over and in parallel with the plates. For testing, input (priming) voltages from 5 to $30 \mathrm{~V}$ were applied. In this paper, only results for $20 \mathrm{~V}$ are presented as they are indicative for the whole voltage range. It is noted that, as expected, higher input voltage gives higher output voltage pulses.

Given the low value of the capacitance at the discharging position of the rod (less than $2 \mathrm{pF}$ ), a buffer circuit was used to provide high input resistance and low capacitance of the measuring system. The complete circuit is shown in Fig. 13. A resistor of $R_{\text {in }}=10 \mathrm{k} \Omega$ is used at the input to prevent high current flow during any unwanted short-circuiting. At the output, a simple, negative feedback operational amplifier configuration is used, with $R_{\text {out }}=10 \mathrm{M} \Omega$ and $R_{f}=1 \mathrm{M} \Omega$, corresponding to a gain of 0.1 . The operational amplifier was an Analog Devices OP249, chosen for high slew rage (22 $\mathrm{V} / \mu \mathrm{s})$ to meet the expected pulse characteristics. The voltage at the output of the buffering circuit was measured using a 10:1 probe and a digital oscilloscope.

A typical output pulse is shown in Fig. 14. A peak output voltage of $48 \mathrm{~V}$ is observed. This value corresponds to a voltage gain of 2.4, which is substantial but not optimal, as the capacitance measurements in Fig. 10 suggest an output to input voltage ratio of 4 . This gain loss may be attributed to charge losses through the $\mathrm{SiO}_{2}$ during the rolling of the rod and to measurement induced losses during pulse capturing by the buffering circuit.

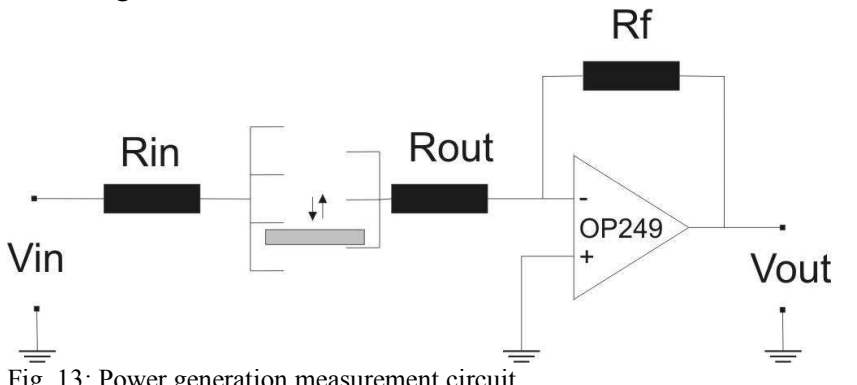

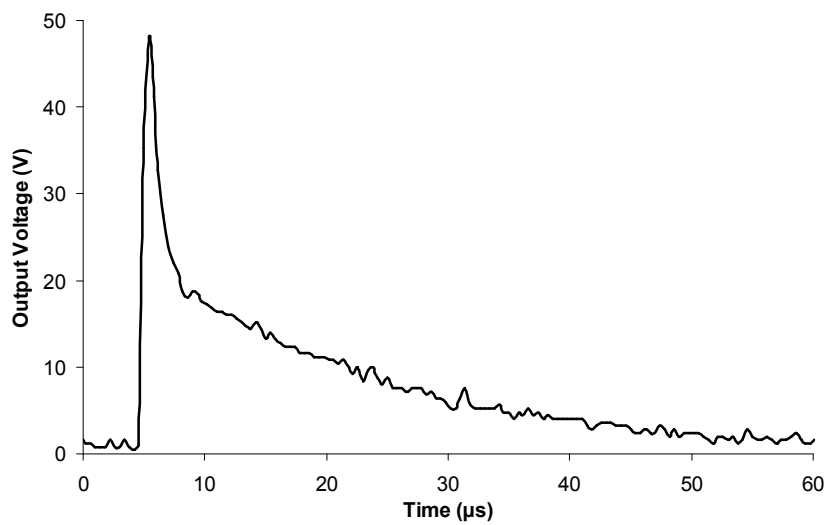

Fig. 14: Typical output voltage pulse of the electrostatic generator.

Taking $C_{\min }$ as $2 \mathrm{pF}$, this corresponds to about $2 \mathrm{~nJ}$ per pulse, or $12 \mathrm{~nJ}$ for a full motion cycle with 3 charge-discharge cycles in each direction. Microwatt power levels are the likely minimum requirement for small electronic devices such as sensors; for input frequencies above $100 \mathrm{~Hz}$ (e.g. machine motion) this is already achievable at such output levels, but more than an order of magnitude increase will be needed for body motion applications, where the frequencies are a few $\mathrm{Hz}$ or less. This will require higher capacitance values $C_{\max }$ and $C_{\text {min }}$ if significantly increased input and output voltage levels are to be avoided.

During measurements, the produced output power is stored as charge at the rod-plate capacitor. The discharge time is much shorter than the duration of contact between rod and output pad ( $>1 \mathrm{~ms}$ ) [12]. Two discharge regions with different time constants are visible in Fig. 14. In order to investigate the origin of these two discharge mechanisms, output measurements for different values of the resistor $R_{\text {out }}$ were performed. The results are shown in Fig. 15. The time constants for both discharge regions were extracted from such pulses and are presented in Table I.

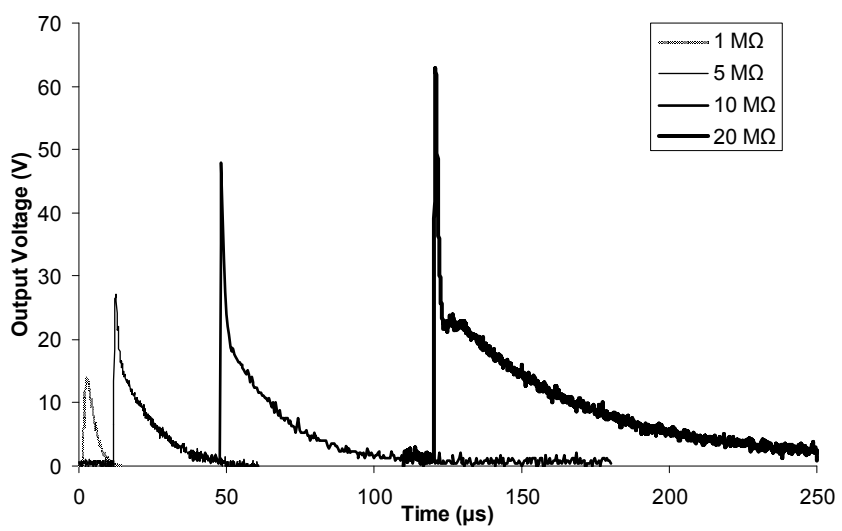

Fig. 15: Output voltage pulses of the generator, for an input voltage of $20 \mathrm{~V}$ and for different values of the measuring circuit input resistance $R_{i n}$. 
TABLE I

OUTPUT DISCHARGE TIME CONSTANTS

\begin{tabular}{ccc}
\hline \hline $\begin{array}{c}\mathrm{R}_{\text {out }} \\
(\mathrm{M} \Omega)\end{array}$ & $\begin{array}{c}\text { Short discharging } \\
\mathrm{RC}(\mu \mathrm{s})\end{array}$ & $\begin{array}{c}\text { Long discharging } \\
\mathrm{RC}(\mu \mathrm{s})\end{array}$ \\
\hline 1 & 4 & 4 \\
5 & 4 & 11 \\
10 & 4 & 22 \\
20 & 3 & 51 \\
\hline \hline
\end{tabular}

The short discharging time constant is independent of $R_{\text {out }}$, and can be explained as charge sharing with the parasitic capacitance between the discharge contacts and earth at the moment of contact. The level of voltage drop between the peak and the commencement of the slow discharge, typically about half the peak, suggests a magnitude of parasitic capacitance on the order of $C_{m i n}$, i.e. a few $\mathrm{pF}$. This charge sharing effect should be nearly instantaneous, and the observed rise and fall times are thus limited by the speed of the operational amplifier circuit. On the other hand, the longer discharge time is approximately proportional to $R_{\text {out }}+R_{f}$. and thus corresponds well to discharge of $C_{\min }$ into the output of the op-amp, for a discharge capacitance value of $3 \pm 1 \mathrm{pF}$. This can be more clearly seen in Fig. 16, where the discharge waveforms are plotted on a log scale. The results are in agreement with the capacitance measurements presented in Fig. 10, showing a capacitance of $2 \mathrm{pF}$ at the output position.

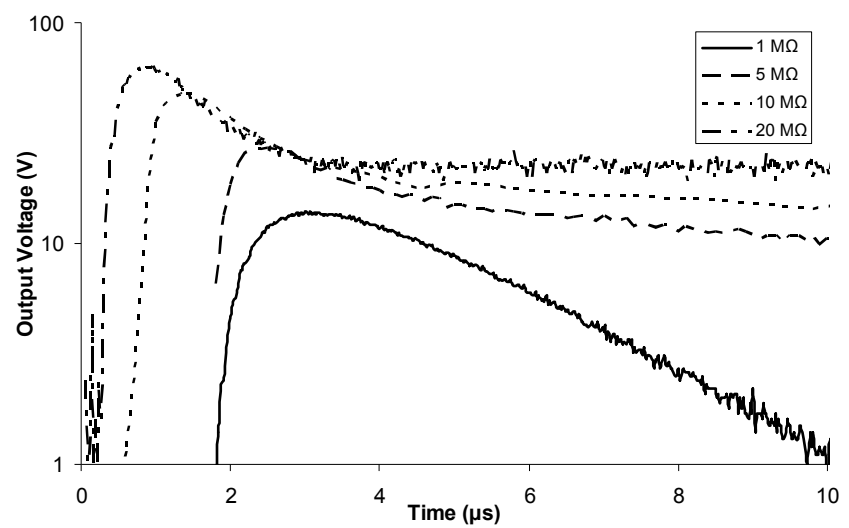

Fig. 16: Output voltage pulses of Fig. 15, replotted on a logarithmic scale

In common with previous electrostatic generators, the device reported here provides power in short output pulses. These pulses need to be transformed efficiently to a usable form, i.e a dc voltage. This requirement has been addressed in detail for parallel-plate constant charge energy harvesters [12], and the results are equally applicable here. A suitable conversion/regulation circuit, using a half-bridge step-down power converter, is shown in Fig. 17.

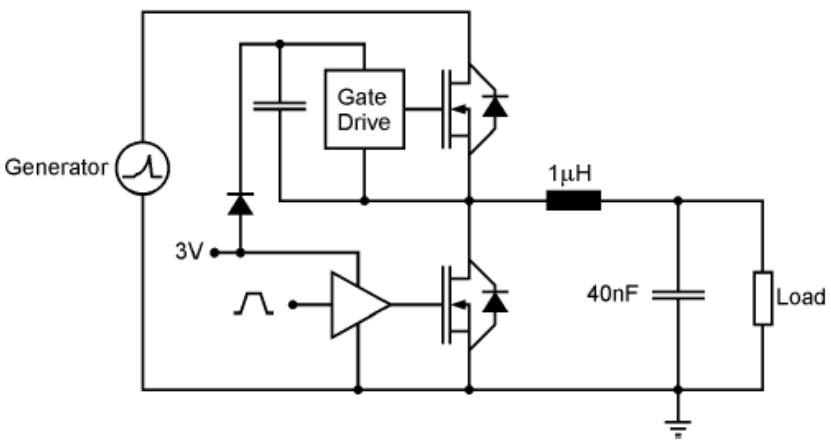

Fig. 17: A half-bridge step-down power converter, after [12].

The electrostatic generator for which this converter was designed [7] produced very high output pulse voltages $(>100$ V). Consequently a novel MOSFET design was required for the switching transistors in the circuit, in order to provide the unusual combination of requirements, i.e. high blocking voltage, low channel resistance, and low input capacitance. In contrast, the present device relaxes these requirements somewhat, as the distribution of the output power of one period of rod motion to a number of output pulses reduces the output amplitudes. This difference may simplify the design and fabrication of the power converter required for the commercial application of the rolling rod electrostatic microgenerator.

\section{CONCLUSIONS}

In this paper, a novel implementation of the recently proposed rolling rod electrostatic microgenerator was presented, and successful operation of the device was demonstrated. The experimental results were compared with simulations and it was shown that increase of capacitance, and hence power generation, is possible by reducing the air gap between rod and dielectric.

Significant further development is required for application of this MEMS energy harvesting device. Higher capacitance values must be achieved, through reduced dielectric thickness and improved rod-surface contact precision, so as to increase the energy per pulse. Power conversion circuits satisfying the requirements of high voltage handling, high efficiency and minimal parasitics must be integrated with the device. A packaging solution is needed which can constrain the rolling proof mass in its correct trajectory without impeding its motion. However, the feasibility of this novel device concept has been demonstrated, and offers the potential for a MEMS scale motion energy harvester, along with the ability to achieve substantial proof mass size and displacement range without the high cost processes of through-wafer machining, or integrated suspensions.

\section{REFERENCES}

[1] J. A. Paradiso and T. Starner, "Energy scavenging for mobile and wireless electronics," Pervasive Computing, IEEE, vol. 4, pp. 18-27, 2005.

[2] S. Roundy, P. K. Wright, and J. M. Rabaey, Energy Scavenging for Wireless Sensor Networks, 1st ed. Boston, Massachusetts: Kluwer Academic Publishers, 2003.

[3] P. D. Micheson, T. Sterken, C. He, M. E. Kiziroglou, E. M. Yeatman and R. Puers, "Electrostatic microgenerators", Measurement and 
control, vol. 41, 114-119, 2008

[4] S. Meninger, J. O. Mur-Miranda, R. Amirtharajah, A. P. Chandrakasan and J. H. Lang, .Vibration-to-Electric Energy Conversion,. IEEE Trans. Very Large Scale Integration (VLSI) Systems, vol. 9, no. 1, pp. 64-76, 2001

[5] S. Roundy, P. K. Wright, and K. S. Pister, .Micro-Electrostatic Vibration-to-Electricity Converters,. in 2002 ASME International Mechanical Engineering Congress and Exposition, New Orleans, Louisiana, USA, November 2002.

[6] T. Sterken, P. Fiorini, K. Baert, K. Puers, and G. Borghs, .An ElectretBased Electrostatic $\mu$-Generator, Proc. 12th International Conference on Solid State Sensors, Actuators and Microsystems (Transducers), Boston, p. 1291, June 2003,.

[7] P. Miao, P. D. Mitcheson, A. S. Holmes, E. M. Yeatman, T. C. Green, and B. H. Stark, "MEMS inertial power generators for biomedical applications," Microsystem Technologies, vol. 12, pp. 1079-1083, 2006.

[8] B. C. Yen and J. H. Lang, "A variable capacitance vibration-to-electric energy harvester," IEEE Trans. Circ. And Systems I, vol. 53 (2), pp. 288-295, 2006

[9] P. D. Mitcheson, T. C. Green, E. M. Yeatman, and A. S. Holmes, "Architectures for vibration-driven micropower generators," Journal of Microelectromechanical Systems, vol. 13, pp. 429-440, 2004.

[10] V. R Challa, M. G. Prasad, Y. Shi and F. T. Fisher, "A vibration energy harvesting device with bidirectional resonance frequency tunability," Smart Mater. Struct. vol. 17, 015035, 2008.

[11] M. E. Kiziroglou, C. He and E. M. Yeatman, "Electrostatic energy harvester with external proof mass," Proc. of Power MEMS, p. 117, Freiburg 28-29 Nov. 2007.

[12] B. H. Stark, P. D. Mitcheson, P. Miao, T. C. Green, E. M. Yeatman and A. S. Holmes, "Power Processing Issues for Micro-Power Electrostatic Generators,", 35th Annual IEEE Power Electronics Specialists Conference Aachen, Germany, 2004

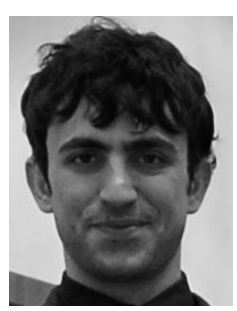

Michail E. Kiziroglou obtained his diploma in electrical and computer engineering from Aristotle University of Thessaloniki, Greece, in 2000 and his master in microelectronics and nanoelectronics from Democritus University of Thrace, Greece, in 2003. He holds a Ph.D. in microelectronics and spintronics awarded by the University of Southampton in 2007. He is currently a researcher with the optical and semiconductor devices group of Imperial College London. His research interests include novel microelectromechanical systems, semiconductor integration and nanoelectronic devices.

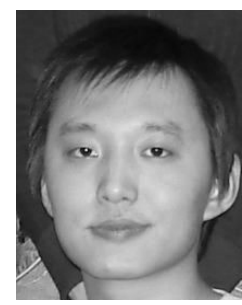

Cairan He received his B.S. degree from the Electrical and Electronic Engineering Department, Imperial College London in 2006. He is currently a Ph.D. student with the Optical and Semiconductor Devices group of Imperial College London. His research interests include microelectromechanical systems and energy scavenging for wireless sensor nodes.

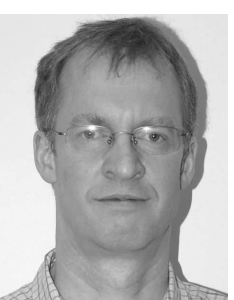

Eric M. Yeatman obtained his B.Sc from Dalhousie University, Canada, in 1983, and his Ph.D. from Imperial College London in 1989. Since then he has been a member of academic staff in the college's Electrical and Electronic Engineering Department, Optical and Semiconductor Devices Group, currently as Professor of Microengineering and Deputy Head of Group. His research interests include micromechanical actuators and generators, microstructures for optical and radio frequency applications, and technologies for pervasive sensing. 